\title{
Local sensitivity analysis of cardiovascular system parameters
}

\author{
R. Gul ${ }^{1} \&$ S. Bernhard ${ }^{1,2}$ \\ ${ }^{1}$ Fachbereich Mathematik, Freie Universität Berlin, Germany \\ ${ }^{2}$ Department of Electrical Engineering and Information Technology, \\ Pforzheim University, Germany
}

\begin{abstract}
Cardiovascular disease is one of the major problems in today's medicine and the number of patients increase worldwide. To treat these types of diseases, prior knowledge about function and dysfunction of the cardiovascular system is essential for identifying the disease in an early stage.

Mathematical modeling is a powerful tool for prediction and investigation of the cardiovascular system. It has been shown, that the Windkessel model, drawing an analogy between electrical circuits and fluid flow, is an effective method to model the human cardiovascular system. The aims of this work are the derivation of a computational cardiovascular model for the arm arteries, and to analyze the behavior of the vascular network structure by parameter sensitivity analysis.

Sensitivity analysis is essential for parameter estimation and simplification of cardiovascular models. In optimal experiment design (OED) sensitivity analysis is used to construct experiments and corresponding models that allow the interpretation of cardiovascular measurements in an effective manner. In this paper we have applied sensitivity analysis to a linear elastic model of the arm arteries to find sensitive parameters and their confidence intervals that guide us to the estimation of cardiovascular network parameters. To calculate the percentage effect on the measurable state variables pressure and flow, with respect to percentage change in cardiovascular input parameters, we use norms. This method allows us to quantify and verify results obtained by sensitivity analysis.

The sensitivities with respect to flow resistance, arterial compliance and flow inertia, reveal that the flow resistance and diameter of the vessels are the most
\end{abstract}


sensitive parameters. Those parameters play a key role in diagnoses of severe stenosis and aneurysms. In contrast, wall thickness and elastic modulus are found to be less sensitive.

Keywords: computational cardiovascular model, cardiovascular parameters, sensitivity analysis, Windkessel model.

\section{Introduction}

With growing interest in the prediction and diagnosis of cardiovascular diseases, different mathematical models have been developed and applied. Windkessel models (electrical analogy to fluid flow) have shown to be an effective approach in modeling the human cardiovascular system [1-4]. Westerhof et al. [3] studied the design, construction and evaluation of an electrical model. Quarteroni et al. [1] introduced a multiscale approach, where local and systemic models are coupled at a mathematical and numerical level. He also introduced the Windkessel models for different inlet and outlet conditions.

Within the Windkessel model the hemodynamic state variables (pressure $(p)$ and flow $(q)$ ), are interrelated to the model parameters like elastic modulus $(E)$, vessel length $(l)$ and diameter $(d)$, wall thickness $(h)$, the density of blood $(\rho)$ and the network structure. Provided that the model parameters estimated correspond to the cardiovascular measurements, the Windkessel model is a good way to study vascular parameters, which are difficult to measure directly.

The basis for robust parameter estimation is on the one hand an optimal experimental measurement setup and on the other hand the development of models that describe the hemodynamic state variables in a set of relevant parameters that can be estimated with high accuracy. The design consists of several logical steps, dealing with questions like:

- How does the optimal measurement setup to identify structural vascular parameters look like?

- What are the parameters, variables and experimental measurement locations within a clinical setting?

- Which vascular system parameters are most influential on the hemodynamic state variables pressure and flow?

- Which vascular system parameters are insignificant and may be fixed or eliminated?

Sensitivity analysis is a powerful approach to find sensitive and therefore important cardiovascular system parameters. The important parameters are further used to design the measurement setup and to modify the computational model for further analysis. Sato et al. [5] studied the effects of compliance, volume and resistance on cardiac output using sensitivity analysis. Yu et al. [6] used parameter sensitivity to construct a simple cardiovascular model. Leguy et al. [7] applied global sensitivity analysis on the arm arteries and showed that the elastic modulus is most sensitive parameter, while arterial length is a less sensitive parameter. In optimal experimental design the information matrix (like, fisher information 
matrix (FIM)) is used for the set of parameters. This information matrix depends on parameter sensitivity analysis.

The methods developed in this paper, are seen as the first step towards cardiovascular system identification from cardiovascular measurements. Within this work we derive a computational cardiovascular model for the arm arteries by using the Windkessel approach. In a first instance we apply local sensitivity analysis to study the effects of cardiovascular parameters on the hemodynamic state variables. Finally we will apply the concept of norms to quantify and compare our results.

\section{Derivation of the model equations}

Under the assumption that the arterial tree is decomposed into short arterial segments of length $l$ with a constant circular cross-section and linear elastic wall behaviour, the following one dimensional flow equations can be derived from the linearized Navier-Stokes equation, the equation of continuity and the shellequation for thin walled, linear elastic tubes $[1,8]$

$$
\begin{aligned}
& -\frac{\partial p}{\partial x}=\mathcal{R} q+\mathcal{L} \frac{\partial q}{\partial t}, \\
& -\frac{\partial q}{\partial x}=\frac{p}{\mathcal{Z}}+C \frac{\partial p}{\partial t} .
\end{aligned}
$$

Within these equations the state variables are the inflow $q$ and the relative pressure $p$. The viscosity and inertial forces of the blood flow are described by the viscous flow resistance $\mathcal{R}$ and blood inertia $\mathcal{L}$ per unit length respectively. The elastic properties of the wall are modeled by a compliance $C$ per unit length, while the outflow is modeled by a leakage $\frac{1}{Z}$ per unit length [8]. Integration of the two partial differential eqns. (1) and (2) along flow axis leads to a system of equations (3) commonly used to describe electrical circuits (see Figure 1). In this type of model each segment of the arterial system is described by a set of two equations that are known as three element Windkessel equations. Here $\left(p_{\text {in }}, q_{\text {out }}\right)$ are the boundary conditions for non-terminal nodes. To model a mean venous pressure with a value of $15 \mathrm{mmHg}$ for terminal nodes the equation system is setup by including an additional terminal resistance $Z$ and using boundary conditions $\left(p_{\text {in }}, p_{\text {out }}\right)$. The matrix form of the Windkessel eqns. with boundary conditions is

$$
\frac{d X}{d t}=A X+B
$$

For non-terminal segments (Figure $1:$ left) $\mathcal{X}=\left(q_{\text {in }}, p_{\text {out }}\right)^{T}$

$$
A=\left(\begin{array}{cc}
\frac{-R}{L} & \frac{-1}{L} \\
\frac{1}{C} & 0
\end{array}\right), \quad B\left(p_{\text {in }}, q_{\text {out }}\right)=\left(\begin{array}{c}
\frac{p_{\text {in }}}{L} \\
-\frac{q_{\text {out }}}{C}
\end{array}\right)
$$


For terminal segments (Figure 1:right) $X=\left(q_{\text {in }}, q_{\text {out }}\right)^{T}$

$$
A=\left(\begin{array}{cc}
\frac{-R}{L} & \frac{-1}{L} \\
\frac{1}{C} & -\frac{1}{Z C}
\end{array}\right), \quad B\left(p_{\text {in }}, p_{\text {out }}\right)=\left(\begin{array}{c}
\frac{p_{\text {in }}}{L} \\
-\frac{p_{\text {out }}}{Z C}
\end{array}\right)
$$

The electrical parameters for $i$-th segment in the arterial tree are related to the physiological parameters of the fluid and vessel wall by:

$$
R_{i}=\frac{8 v l}{\pi r^{4}}, \quad L_{i}=\frac{\rho l}{\pi r^{2}}, \quad C_{i}=\frac{2 \pi r^{2} l}{E h} .
$$

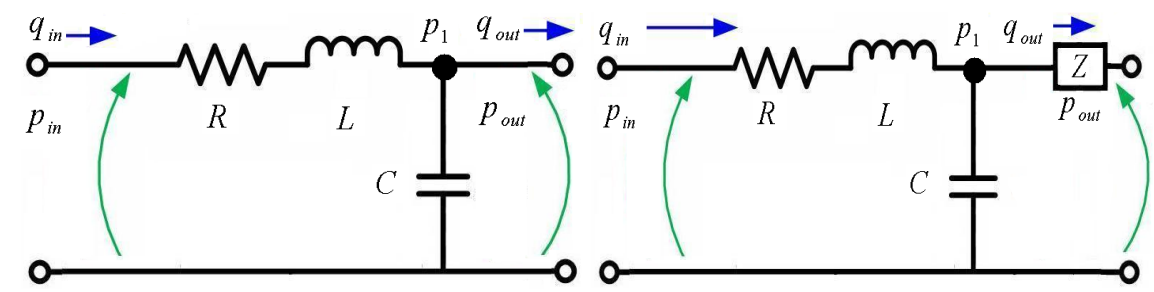

Figure 1: Linear elastic model for fluid flow in nonterminal vessel segments (left) and for terminal vessel segments (right).

\subsection{Vascular model of the arm arteries}

\subsubsection{Network structure and model equations}

Each segment of arm arteries in a network structure as given in Figure 2 is represented by an electrical circuit as shown in Figure 1.

Applying Kirchhoff's current and voltage laws to the arterial structure given in Figure 2, with number of terminals $N_{t}=3$ and number of segments $N_{s}=15$, we obtain a system of coupled ordinary differential equations for pressure and flow. Flow equations:

$$
\begin{aligned}
& \dot{q}_{1}=\frac{p_{\text {in }}-p_{1}-R_{1} q_{1}}{L_{1}}, \quad \dot{q}_{2}=\frac{p_{1}-p_{2}-R_{2} q_{2}}{L_{2}}, \quad \dot{q}_{3}=\frac{p_{2}-p_{3}-R_{3} q_{3}}{L_{3}} \\
& \dot{q}_{4}=\frac{p_{3}-p_{4}-R_{4} q_{4}}{L_{4}}, \quad \dot{q}_{5}=\frac{p_{4}-p_{5}-R_{5} q_{5}}{L_{5}}, \quad \dot{q}_{6}=\frac{p_{5}-p_{6}-R_{6} q_{6}}{L_{6}} \\
& \dot{q}_{7}=\frac{p_{6}-p_{7}-R_{7} q_{7}}{L_{7}}, \quad \dot{q}_{8}=\frac{p_{7}-p_{8}-R_{8} q_{8}}{L_{8}}, \quad \dot{q}_{9}=\frac{p_{8}-p_{9}-R_{9} q_{9} .}{L_{9}} \\
& \dot{q}_{10}=\frac{p_{9}-p_{10}-R_{10} q_{10}}{L_{10}}, \dot{q}_{11}=\frac{p_{6}-p_{11}-R_{11} q_{11}}{L_{11}}, \dot{q}_{12}=\frac{p_{11}-p_{12}-R_{12} q_{12}}{L_{12}} \\
& \dot{q}_{13}=\frac{p_{11}-p_{13}-R_{13} q_{13}}{L_{13}}, \dot{q}_{14}=\frac{p_{13}-p_{14}-R_{14} q_{14}}{L_{14}}, \dot{q}_{15}=\frac{p_{14}-p_{15}-R_{15} q_{15}}{L_{15}}
\end{aligned}
$$


Pressure equations:

$$
\begin{aligned}
& \dot{p}_{1}=\frac{q_{1}-q_{2}}{C_{1}}, \quad \dot{p}_{2}=\frac{q_{2}-q_{3}}{C_{2}}, \quad \dot{p}_{3}=\frac{q_{3}-q_{4}}{C_{3}}, \quad \dot{p}_{4}=\frac{q_{4}-q_{5}}{C_{4}} \\
& \dot{p}_{5}=\frac{q_{5}-q_{6}}{C_{5}}, \quad \dot{p}_{6}=\frac{q_{6}-q_{11}-q_{7}}{C_{6}}, \quad \dot{p}_{7}=\frac{q_{7}-q_{8}}{C_{7}}, \quad \dot{p}_{8}=\frac{q_{8}-q_{9}}{C_{8}} \\
& \dot{p}_{9}=\frac{q_{9}-q_{10}}{C_{9}}, \quad \dot{p}_{10}=\frac{q_{10}-\left(p_{10}-p_{\text {out }}\right) / Z_{1}}{C_{10}}, \quad \dot{p}_{11}=\frac{q_{11}-q_{12}-q_{13}}{C_{11}} \\
& \dot{p}_{12}=\frac{q_{12}-\left(p_{12}-p_{\text {out }}\right) / Z_{2}}{C_{12}}, \quad \dot{p}_{13}=\frac{q_{13}-q_{14}}{C_{13}}, \quad \dot{p}_{14}=\frac{q_{14}-q_{15}}{C_{14}} \\
& \dot{p}_{15}=\frac{q_{15}-\left(p_{15}-p_{\text {out }}\right) / Z_{3}}{C_{15}}
\end{aligned}
$$

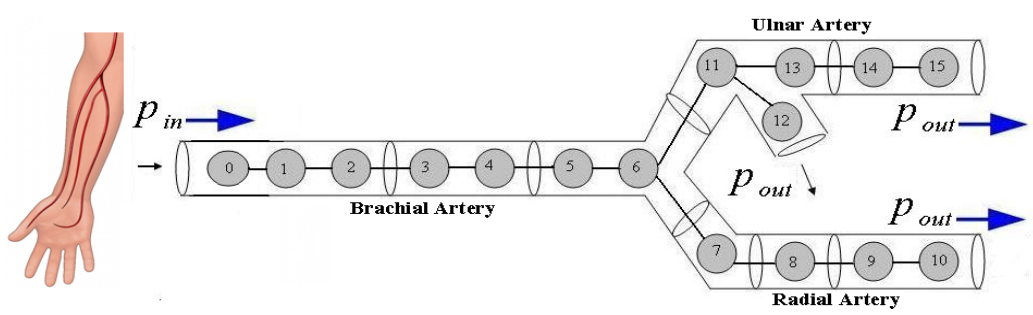

Figure 2: Simplified anatomy of the arm arteries (left) and model geometry with $N_{s}=15$ and $N_{t}=3$ (right).

\subsection{State-space representation}

The state-space representation is a useful approach to describe the dynamics in arterial networks efficiently [9]. In state-space form, we have a system of two equations: an equation for determining state $x_{t}$ of the system (state equation), and another equation to describe the output $y_{t}$ of the system (observation equation). The matrix form can be written as

$$
\begin{aligned}
& \dot{x}_{t}=A x_{t-1}+B u_{t}, \\
& y_{t}=C x_{t}+D u_{t} .
\end{aligned}
$$

Here $x_{t}$ is the state vector of the system, $u_{t}$ the input vector and $y_{t}$ the observation vector. The dynamics of the system is described by the state dynamics matrix $A \in M(n \times n)$. The input matrix $B \in M(n \times i)$ specifies the time dependency of the in- and outflow boundary values and the observation matrix $C \in M(m \times n)$ defines the observation locations within the state-space system, i.e. the nodal location in 
the network. Here $m$ denotes the number of observations. Finally the input to observation matrix $D \in M(m \times i)$ adds the influence of the input vectors to the observation vectors. Besides the computational advantage the state-space form allows the integration of experimental measurements (observations) into the model building process. This step is essential for the adjacent model parameter estimation from experimental measurements, that we have planned in a future study.

In the current study parameter values were taken from the literature [4]. The state vector $x_{t}$ contains the flow and pressure functions at all network locations, whereas the output vector $y_{t}$ contains the flow and pressure at selected nodes $i$. For a $m=4$ dimensional observation vector, the output vector is e.g. $y(t)=$ $\left(q_{5}(t), p_{5}(t), q_{6}(t), p_{6}(t)\right)^{T}$ where $y \in R^{m}$ at nodes 5 and 6 . The state-space system for the arm artery given in Figure 2 is then defined by

$$
\begin{aligned}
& A_{i j}=\left\{\begin{array}{cl}
\frac{-R_{\frac{i+1}{2}}}{L_{\frac{i+1}{2}}} & i=1,3,5, \ldots, 29, j=i \\
\frac{1}{C_{\frac{i}{2}}} & i=2,4,6, \ldots, 30, j=i-1 \\
\frac{-1}{L_{\frac{i+1}{2}}} & i=1,3,5, \ldots, 29, j=i+1 \\
\frac{1}{L_{\frac{i+1}{2}}} & i=3,5,7, \ldots, 29, j=i-1 \text { and } i \neq 21,25 \\
& \text { also for } i=21, j=12 \text { and } i=25, j=22 \\
\frac{-1}{C_{\frac{i}{2}}} & i=2,4,6, \ldots, 30, j=i+1 \text { and } i \neq 20,24 \\
\frac{-1}{Z_{k} C_{\frac{i}{2}}} & k=1,2,3, i=20,24,30, j=i \\
0 & \text { otherwise }
\end{array}\right. \\
& B_{i j}=\left\{\begin{array}{ll}
\frac{1}{L_{i}} & i=j=1 \\
\frac{1}{Z_{j-1} C_{\frac{i}{2}}} & i=20, j=2 \\
\frac{1}{Z_{j-1} C_{\frac{i}{2}}} & i=24, j=3 \\
\frac{1}{Z_{j-1} C_{\frac{i}{2}}} & i=30, j=4 \\
0 & \text { otherwise }
\end{array} \quad C_{i j}= \begin{cases}1 & i=1,2,3,4, j=i+8 \\
0 & \text { otherwise }\end{cases} \right.
\end{aligned}
$$

and $D_{i j}=0$.

\section{Methods of local sensitivity analysis}

To understand the interdependence of the state variables and the parameters of the cardiovascular model, it is not enough to compute a solution, but also to quantify the sensitivity of the model parameters. Sensitivity analysis is a useful tool to quantify the variation in state variables at different nodes in the vascular network caused by a change in model parameters. Due to the interdependence of 
the electrical analog parameters $R C L$, we also discuss the linear independent basis parameters $E L d h$. Further, we are interested in how the node location within the network influences the results. Therefore we discuss three different scenarios. The sensitivity results obtained are compared to the 2-norm of the distance vector of the state variables of two time series.

\subsection{Sensitivity with respect to $E, l, d$ and $h$}

The cardiovascular model consists of a system of ODEs with a parameter set $\theta$ and an initial condition $y_{i}(0)$, given by

$$
\frac{d y}{d t}=f_{i}\left(y_{i}, \theta, t\right) \quad i=1,2, \ldots, n .
$$

In local sensitivity analysis, parameters are varied segmentwise by some portion around a fixed value and the effects of individual perturbations on the observations are studied [10]. Using differential calculus the sensitivity coefficients are

$$
S_{i}=\frac{\partial y_{i}}{\partial \theta}=\lim _{\Delta \theta \rightarrow 0} \frac{y_{i}(\theta+\Delta \theta)-y_{i}(\theta)}{\Delta \theta},
$$

where $y_{i}$ is the $i$ th model output and $\theta$ is the model input parameter. There exists a variety of methods to compute the sensitivity coefficients in eqn. (8), within this work we use the method of external differentiation:

$$
S_{i}=\frac{\partial y_{i}}{\partial \theta} \simeq \frac{y_{i}(\theta+\Delta \theta)-y_{i}(\theta)}{\Delta \theta}
$$

Applied to our network structure, this equation produces a set of two sensitivity time series $S_{i}(t)$ (one for pressure and one for flow) per parameter and per network node (see Figure 3 ).

\subsection{Sensitivity with respect to $R, C$ and $L$}

To find the sensitivity of the electrical analog parameters $R, C$ and $L$ on cardiovascular pressure and flow, we solve eqn. (7) numerically using the CVODES solver, which is a part of SUNDIALS software suit [11,12]. The computational method is also based on external differentiation.

\subsection{Network structure and sensitivity}

To study the influence of the vascular network structure, we use a physiological network structure with identical (non-physiological) Windkessel elements, i.e. the parameters $R_{i}, C_{i}$ and $L_{i}$ are identical for each node. This allows us to analyze the influence of the network structure onto sensitivity values at different node locations. Therefore we compute the nodal sensitivity time series $S_{i}$ for all nodal parameters as described in previous section. For each node, $n$, we obtain two 
sensitivity time series (one for the pressure and one for the flow) per parameter variation. The total number of possible parameter variations is $3 n^{2}$, so we end up with a set of $6 n^{2}$ time series. To reduce the complexity, we average the time dependency by computing the mean of the absolute value of every time series. The $6 n^{2}$ real values are used for further analysis in section 4 . They are displayed into 6 matrices that characterize the sensitivity of pressure and flow in the network structure based on changes in the electrical parameters $R C L$. Each cell in Figure 5 represents the mean absolute value of time series $\left(S_{i}\right)$. Due to the fact that we use the pressure as an input and output boundary condition, the change in pressure with any parameter at all inlet and terminal nodes will be zero.
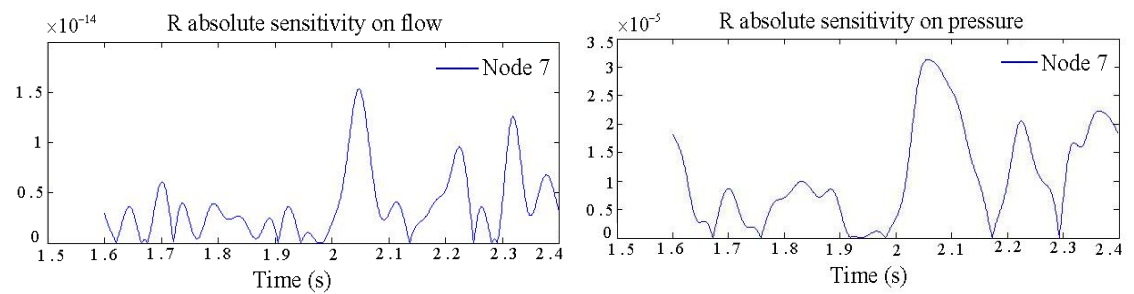

Figure 3: Time series of flow resistance $R$ sensitivity coefficient $S_{i}$ for pressure and flow at node 7 .

\subsection{Sensitivity analysis by using norms}

To obtain a suitable measure for sensitivity we calculated the mean Euclidean distances of observations made in a model with different parameter sets $\theta_{1}, \theta_{2}$.

$$
\left\|\theta_{1}, \theta_{2}\right\|:=\operatorname{mean}_{t \in T} \frac{\left\|y_{i}\left(\theta_{2}, t\right)-y_{i}\left(\theta_{1}, t\right)\right\|_{2}}{\left\|y_{i}\left(\theta_{1}, t\right)\right\|_{2}} \quad i=1,2,3, \ldots, 2 \times N_{s},
$$

\section{Results and discussion}

Our local sensitivity analysis was carried out as described in section 3 . The results are structured into the following sections:

\subsection{Sensitivities with respect to physiological parameters}

The sensitivity for pressure and flow are obtained by a variation of the cardiovascular parameters $E, l, d$ and $h$ of arm arteries by $\pm 10 \%$. From Figure 4 it is directly evident, that diameter and length are most sensitive parameters, while the elastic modulus and wall thickness are comparatively less sensitive parameters. 

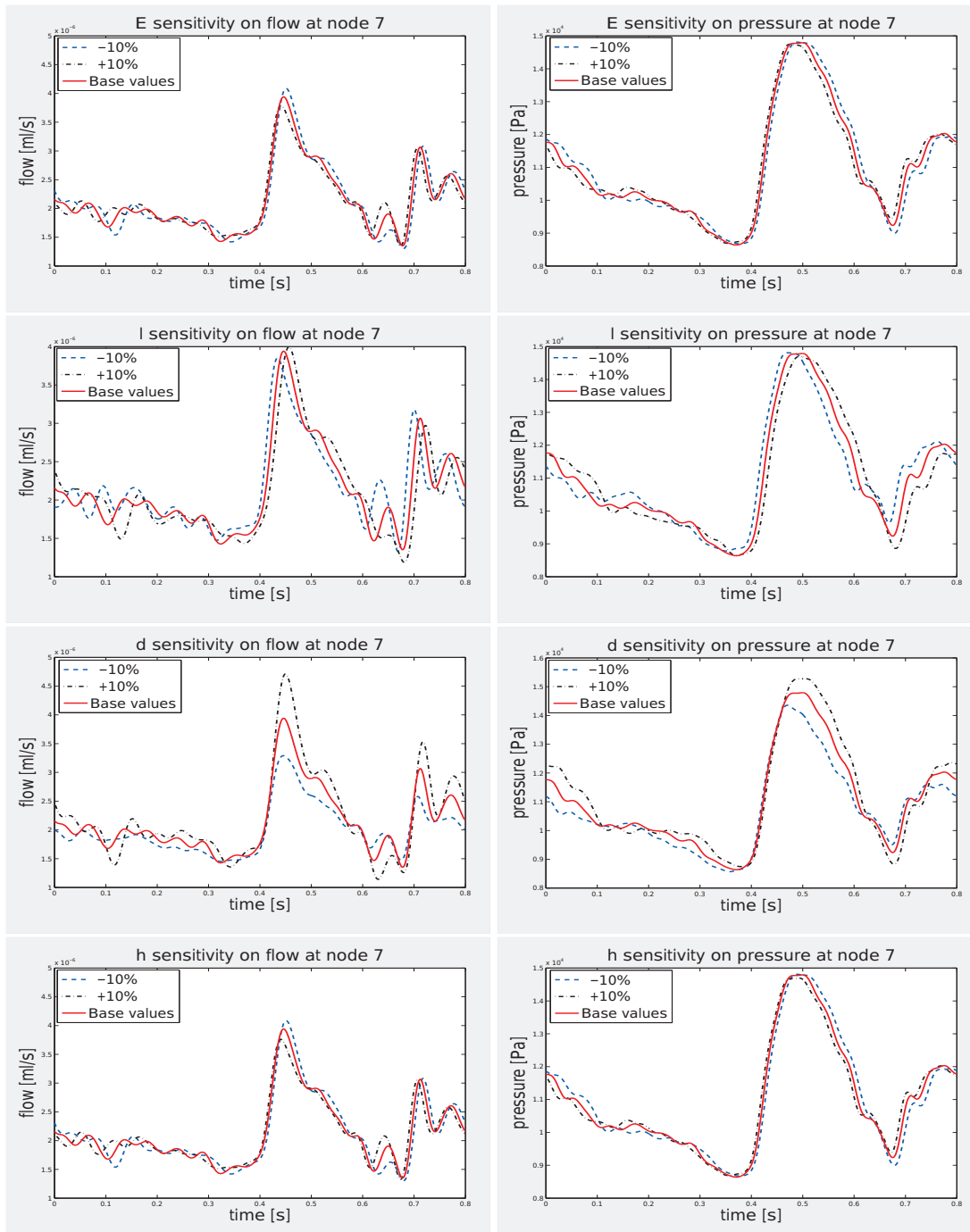

Figure 4: $\pm 10 \%$ change in $E, l, d$ and $h$ in arm artery at node 7 .

\subsection{Sensitivities with respect to fluid dynamical parameters}

The sensitivities w.r.t. $R, C$ and $L$ were calculated by forward sensitivity analysis (FSA) using the SUNDIALS software. The sensitivity pattern obtained by variation of the viscous flow resistance $R$ in any segment of brachial artery (see Figure 5 (top)) indicates a strong local (within brachial artery) influence on flow and has significant global influence on all following nodes of the brachial, ulnar and radial arteries. In contrast, changing $R$ in the parallel association of the ulnar 
and radial arteries have negligible local and global effects, because in parallel arteries the total flow resistance is given by the fraction $\frac{1}{R_{\text {total }}}=\frac{1}{R_{\text {ulnar }}}+\frac{1}{R_{\text {radial }}}$, i.e. due to the increment in total diameter the flow resistance reduces. Physically a change of $R$ in one branch redirects the flow into the other branch while the overall flow is maintained. The sensitivity of flow resistance in parallel branches is thus smaller than in series connections.

In contrast to the flow resistance, the sensitivity of arterial compliance $C$ in brachial part has small influence (locally and globally) on pressure and flow (see Figure 5 (middle)). This can be explained by the fact, that in series segments the total compliance is given by the sum of all segmental compliances in series $C_{\text {total }}=\sum_{i=1}^{6} C_{i}$. The total compliance is larger than the individual compliances, thus a change of $C$ in any node has a small effect on pressure and flow in the whole arm artery. In contrast a variation of the arterial compliance $C$ in the ulnar and radial arteries have a large (local) effect on pressure and large (global) effect on flow especially in the brachial artery.
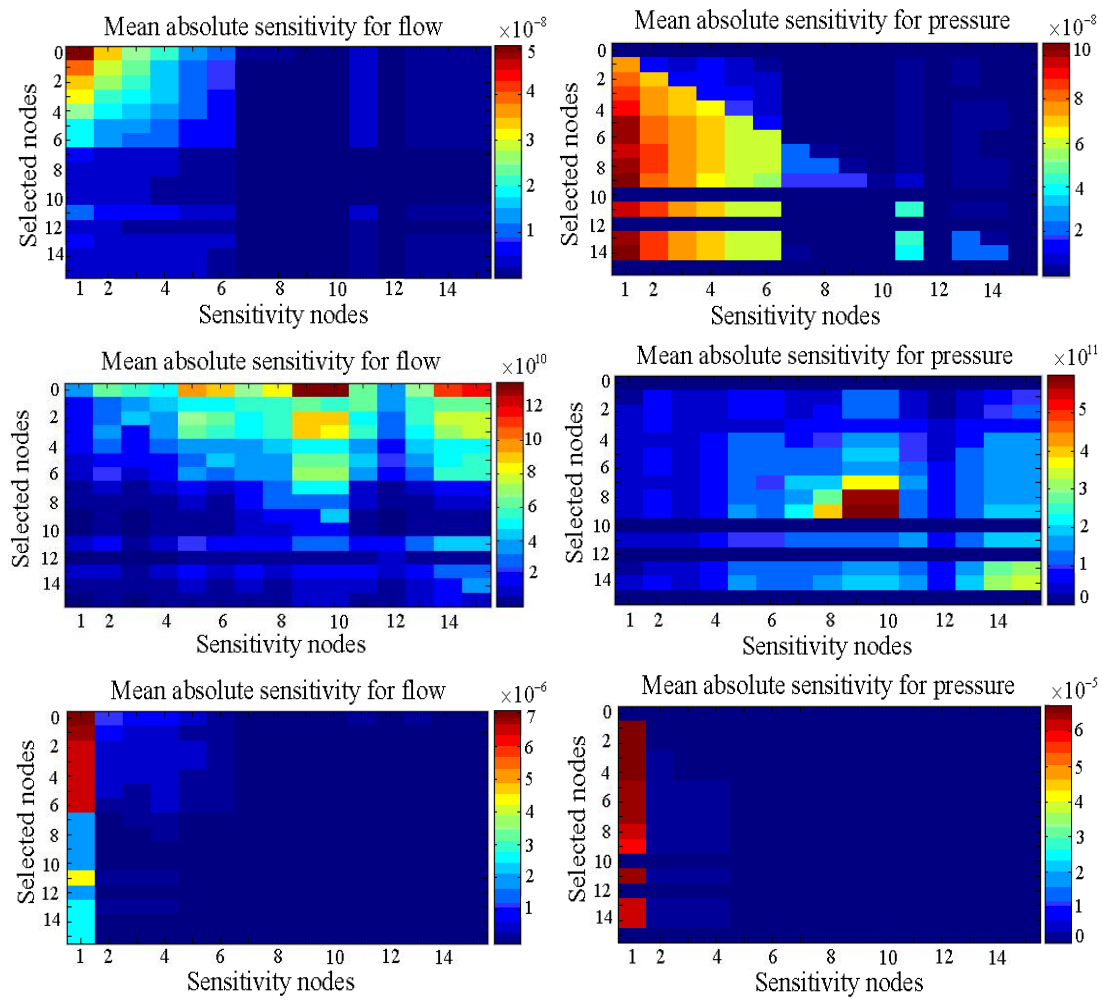

Figure 5: Effects of viscous flow resistance $R$ (top), vessel compliance $C$ (middle) and blood inertia $L$ (bottom) on pressure and flow in the arm arteries. 
From eqn. (4) it is obvious that viscous resistance and blood inertia are inversely related to $r^{4}$ and $r^{2}$ respectively. Which means in large arteries blood inertia plays an important role, while in small arteries viscous resistance is more important. A variation of blood inertia in the first node of the brachial artery has large influence on pressure and flow of all following nodes of the arm arteries (see Figure 5 (bottom)). However, we observe only minor local (within brachial artery) effects on flow when we change $L$ in each segment of the brachial artery. Further, due to the fact that the total inductance $\frac{1}{L_{\text {total }}}=\frac{1}{L_{\text {ulnar }}}+\frac{1}{L_{\text {radial }}}$ reduces at the furcation, the flow and pressure in the ulnar and radial arteries are less sensitive with respect to $L$.

\subsection{Sensitivities with respect to norms}

Finally we compare the results obtained by sensitivity analysis with those obtained by using norms (see Table 1 and 2). We found that the diameter and length of vessel are most influential parameters and that the norm computed for the wall thickness and elastic modulus has identical values.

\section{Conclusion}

The sensitivity analysis is the first step towards the estimation of model parameters from experiments. It identifies the parameters in our model that are important to describe the dynamic behaviour of the system, i.e. it defined the parameters that should be estimated correctly and on the other hand those parameters that are less important. Sensitivity analysis thus allows the design of problem specific experiments, i.e. just gather the information that is required to generate a predictive model that describes the actual health status of a patient. It therefore improves the model quality and thus the ability for diagnosis and prediction in cardiovascular physiology. This finally benefits the medical doctor in decision making.

In this work we applied different methods of sensitivity analysis to a lumped parameter Windkessel model of the arm arteries. The results indicate a strong dependence of the pressure and flow state variables onto a variation in vessel diameter and length. According to the elastic properties and the thickness of the arterial wall, a much lower sensitivity was found.

Further, we have used the concept of norms to compare the variation in state variables according to parameter changes. We found a good agreement to the results obtained by sensitivity analysis.

The methods applied, give satisfactory results if the cardiovascular parameters are independent, in the real scenarios however, they are often interdependent like e.g. the observation of a high correlation between the extension of the elastic walls and the tangential tension caused by transmural pressure. To study these type of effects in a more general way, we plan to apply global sensitivity analysis to a closed loop cardiovascular system model, which deals with variations in many parameters at a time. 


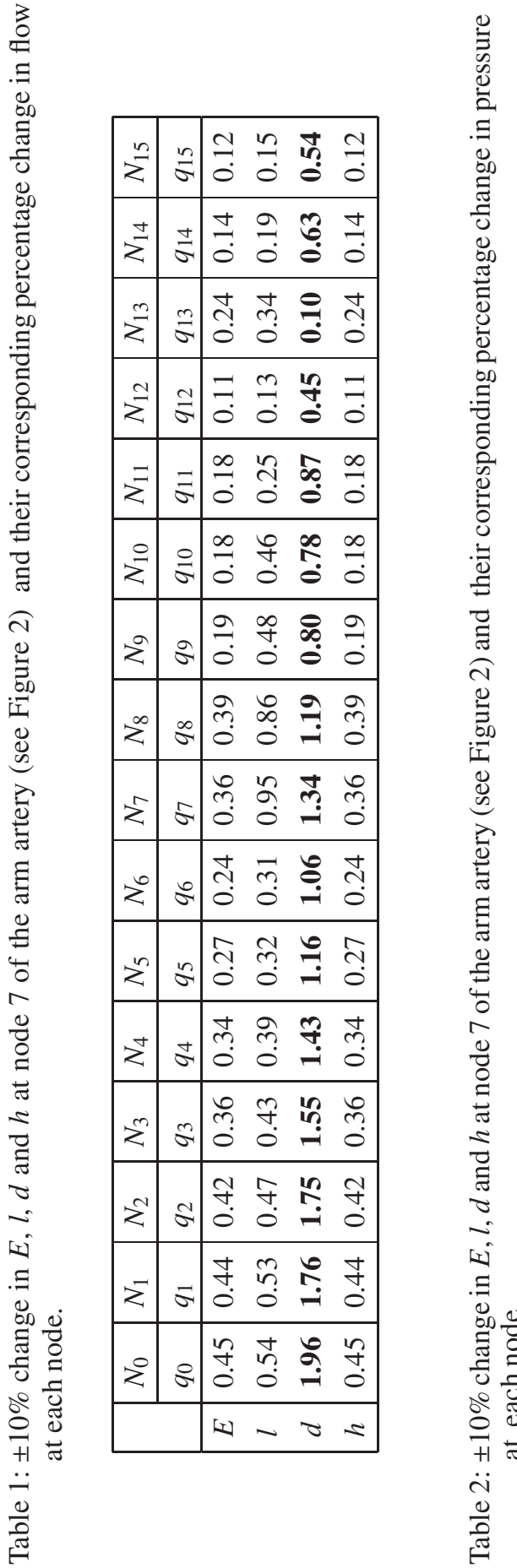

\begin{tabular}{|c|c|c|c|c|c|}
\hline$\sum^{n}$ & $\stackrel{n}{2}$ & * & 0 & $\theta$ & 0 \\
\hline \pm & $\stackrel{ \pm}{2}$ & $\stackrel{0}{\stackrel{0}{0}}$ & $\stackrel{c}{\stackrel{1}{0}}$ & $\stackrel{+}{+}$ & $\stackrel{0}{\stackrel{0}{0}}$ \\
\hline$\sum^{2}$ & $\stackrel{m}{2}$ & $\stackrel{8}{0}$ & $\overline{0}$ & $\stackrel{?}{\stackrel{\theta}{0}}$ & $\stackrel{8}{8}$ \\
\hline 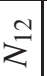 & $\stackrel{2}{2}$ & * & 0 & 0 & 0 \\
\hline & $\Xi$ & $\stackrel{9}{0}$ & $\overline{0}$ & ஸ் & $\stackrel{\circ}{\circ}$ \\
\hline$\frac{0}{z}$ & $\stackrel{\circ}{2}$ & * & 0 & 0 & 0 \\
\hline 2 & ฉ & $\vec{D}$ & $\tilde{n}$ & $\begin{array}{l}\ddot{0} \\
\dot{0}\end{array}$ & $\stackrel{ \pm}{0}$ \\
\hline 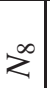 & $\stackrel{\infty}{2}$ & $\frac{m}{0}$ & กै & \begin{tabular}{l}
$\infty$ \\
$i n$ \\
\hdashline \\
0
\end{tabular} & $\stackrel{m}{0}$ \\
\hline & $\Sigma$ & & $\stackrel{\sim}{\tilde{n}}$ & 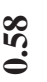 & $\stackrel{m}{0}$ \\
\hline Z & 2 & $\stackrel{\infty}{\infty}$ & $\begin{array}{l}0 \\
0\end{array}$ & ஸे? & $\stackrel{\infty}{\circ}$ \\
\hline n & $\approx$ & $\begin{array}{l}\infty \\
0 \\
0\end{array}$ & $\stackrel{\partial}{\partial}$ & $\ddot{m}$ & $\stackrel{\infty}{\circ}$ \\
\hline \& & \pm & $\stackrel{0}{\circ}$ & $\stackrel{\infty}{\infty}$ & ஸ̃ & $\begin{array}{l}\circ \\
\stackrel{0}{0}\end{array}$ \\
\hline z & $\Omega$ & $\stackrel{n}{0}$ & $\stackrel{0}{\circ}$ & ָூ & $\stackrel{n}{0}$ \\
\hline $\mathcal{\Sigma}$ & 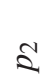 & ڤ్ & $\stackrel{n}{0}$ & $\frac{10}{9}$ & $\stackrel{3}{0}$ \\
\hline$\sum$ & $\overline{2}$ & ర̃ & 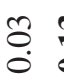 & $\frac{m}{0}$ & 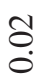 \\
\hline$\sum$ & 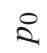 & $*$ & 0 & 0 & 0 \\
\hline & & 仙 & -7 & $\tau$ & $=$ \\
\hline
\end{tabular}




\section{Acknowledgements}

The author would like to thank the HEC/DAAD for their financial support also to Dr. S. Bernhard and Rudolf Huttary for fruitful discussions and encouragement.

\section{References}

[1] Quarteroni, A., Ragni, S. and Veneziani, A., Coupling between lumped and distributed models for blood flow problems. Computing and Visualization in Science. Volume 4, pages 111-124 (2001)

[2] Milisic, V. and Quarteroni, A., Analysis of lumped parameter models for blood flow simulations and their relation with 1D models. Mathematical Modeling and Numerical Analysis (1999)

[3] Westerhof, N., Bosman, F., De Vries, C.J. and Noordergraaf, A., Analog studies of the human systemic arterial tree. Journal of Biomechanics, Volume 2, pages 121-143 (1969)

[4] Noordergraaf, A., Verdouw, P.D. and Boom, H.B.K., The use of an analog computer in a circulation model. Progress in Cardiovascular Diseases. Volume 5 , number 5 (1963)

[5] Sato, T., Yamashiro, S.M., Vega, D. and Grodins, F.S., Parameter sensitivity analysis of a network model of systemic circulatory mechanics. Annals of Biomedical Engineering. Volume 2, pages 289-306 (1974)

[6] Yu, Y.C., Boston, J.R., Simaan, M.A. and Antaki, J.F., Sensitivity analysis of cardiovascular models for minimally invasive estimation of systemic vascular parameters. Proceedings of American Control Conference, San Diago, California, (1999)

[7] Leguy, C.A.D., Bosboom, E.M.H., Belloum, A.S.Z., Hoeks, A.P.G. and van de Vosse, F.N., Global sensitivity analysis of a wave propagation model for arm arteries. Medical Engineering and Physics. Volume 33, pages 1008-1016 (2011)

[8] Jager, Gerad S., Westerhof, N., Noordergraf, A., Oscillatory flow impedance in electrical analog of arterial system: representation of sleeve effect and nonnewtonian properties of blood. Circulation research. Volume XVI, pages 121133 (1965)

[9] Bernhard, S., Al Zoukra, K. and Schütte, C., Statistical parameter estimation and signal classification in cardiovascular diagnosis. Environmental Health and Biomedicine. Volume 15, pages 458-469 (2011)

[10] Zi, Z., Sensitivity analysis approaches applied to systems biology models. IET system biology. Volume 5, issue 6, pages 336-346 (2011).

[11] Hindmarsh, A.C., Brown, P.N., Grant, K.E. et al. SUNDIALS: suite of nonlinear and differential/algebraic equation solvers, ACM Transactions on Mathematical Software. Volume 31, Number 3, pages 363-396 (2005)

[12] Serban, R., Hindmarsh, A.C., CVODES: the sensitivity-enabled ODE solver in SUNDIALS. Proceedings of IDETC/CIE (2005) 